\title{
Pedestrian wind comfort: Feasibility study of criteria homogenisation
}

\author{
A. Sanz-Andres, A. Cuerva
}

One of the aims of COST C14 action is the assessment and evaluation of pedestrian wind comfort. At present there is no general rule available that is applied across Europe. There are several criteria that have been developed and applied in different countries. These criteria are based on the definition of two independent parameters, a threshold effective wind speed and a probability of exceedence of this threshold speed. The difficulty of the criteria comparison arises from the two-dimensional character of the criteria definition. An effort is being made to compare these criteria, trying both to find commonalities and to clearly identify differences, in order to build up the basis for the next step: to try to define common criteria (perhaps with regional and seasonal variations). The first point is to define clearly the threshold effective wind speed (mean velocity definition parameters: averaging interval and reference height) and equivalence between different ways of defining the threshold effective wind speed (mean wind speed, gust equivalent mean, etc.) in comparable terms (as far as possible). It can be shown that if the wind speed at a given location is defined in terms of a probability distribution, e.g. Weibull function, a given criterion is satisfied by an infinite set of wind conditions, that is, of probability distributions. The criterion parameters and the Weibull function parameters are linked to each other, establishing a set called iso-criteria lines (the locus of the Weibull function parameters that fulfil a given criterion). The relative position of iso-criteria lines when displayed in a suitable two-dimensional plane facilitates the comparison of comfort criteria. The comparison of several wind comfort criteria, coming from several institutes is performed, showing the feasibility and limitations of the method. 


\section{Introduction}

The aim of the paper is to show the feasibility of wind comfort criteria comparison as a first step in the process of criteria homogenisation. A summary of some of these criteria is presented in Section 1. The main difficulty in the homogenisation process is the comparison itself, because the comfort criteria are defined by using two parameters, which in principle impedes the possibility of a direct comparison.

Here a method is proposed (Section 2) that allow us to compare the comfort criteria in a rational way, by considering the set of wind climates that fulfil a given criterion, displaying it as a so-called iso-criterion line. Furthermore, in a suitable coordinate set the iso-criteria lines can be plotted as straight lines, which makes comparison even easier, because the relative position of the iso-criteria lines associated with each comfort criteria is easily realized. This method is applied to some of the criteria summarized in Section 1.

In a practical comfort evaluation, the wind climate data are arranged as Weibull probability distribution functions associated with specific wind directions. These probability functions should be combined to obtain the total probability of exceedence. An analytical method to perform such a combination is presented in Section 3, which allows us to define a kind of equivalent local mean wind. The distance from the point representing the equivalent local mean wind and the appropriate iso-criterion comfort give us an idea of the comfort degree.

Finally, main conclusions (Section 3) are summarized and in the Appendix the method explained in Section 3 is justified.

The aim is just to show the feasibility of the criteria comparison, but not to extend the comparison to all the existing criteria. This global comparison could be the subject of an international cooperation with the aim of standardising in some way the existing methods and criteria, and that should also take into account other elements of human perception (air temperature, humidity, acclimatisation, etc.).

\subsection{Background}

In most urban areas an important problem is the achievement of an acceptable wind comfort around the buildings, with aspects that concern the quality of life and the use of the area affected by the buildings, associated to social and economic impacts. To allow for the evaluation of the wind comfort in a practical situation, numerous wind comfort criteria (Davenport, 1972; Melbourne, 1978; Isyumov and Davenport, 1975; Gandemer, 1975) have been developed over the years. The criteria developed by different researchers show relevant differences, due both to the differences in the respective approaches and to the regional conditions of the places where the criteria were developed. Some studies have been carried out to compare the existing criteria (Ratcliff and Peterka, 1990; Koss, 2004). A detailed recent review of the efforts devoted to study the assessment of pedestrian wind comfort and the historical context is given in (Koss, 2004), so it has been considered not nessesary to include a similar review here.

\subsection{Existing criteria}

Wind comfort criteria defined by several institutions participating in COST 14 are shown in Table 1 . These criteria are based on the definition of the probability of exceedence $P_{\text {exc }}$ of a given threshold effective wind speed $V$. According to the threshold effective wind speed 


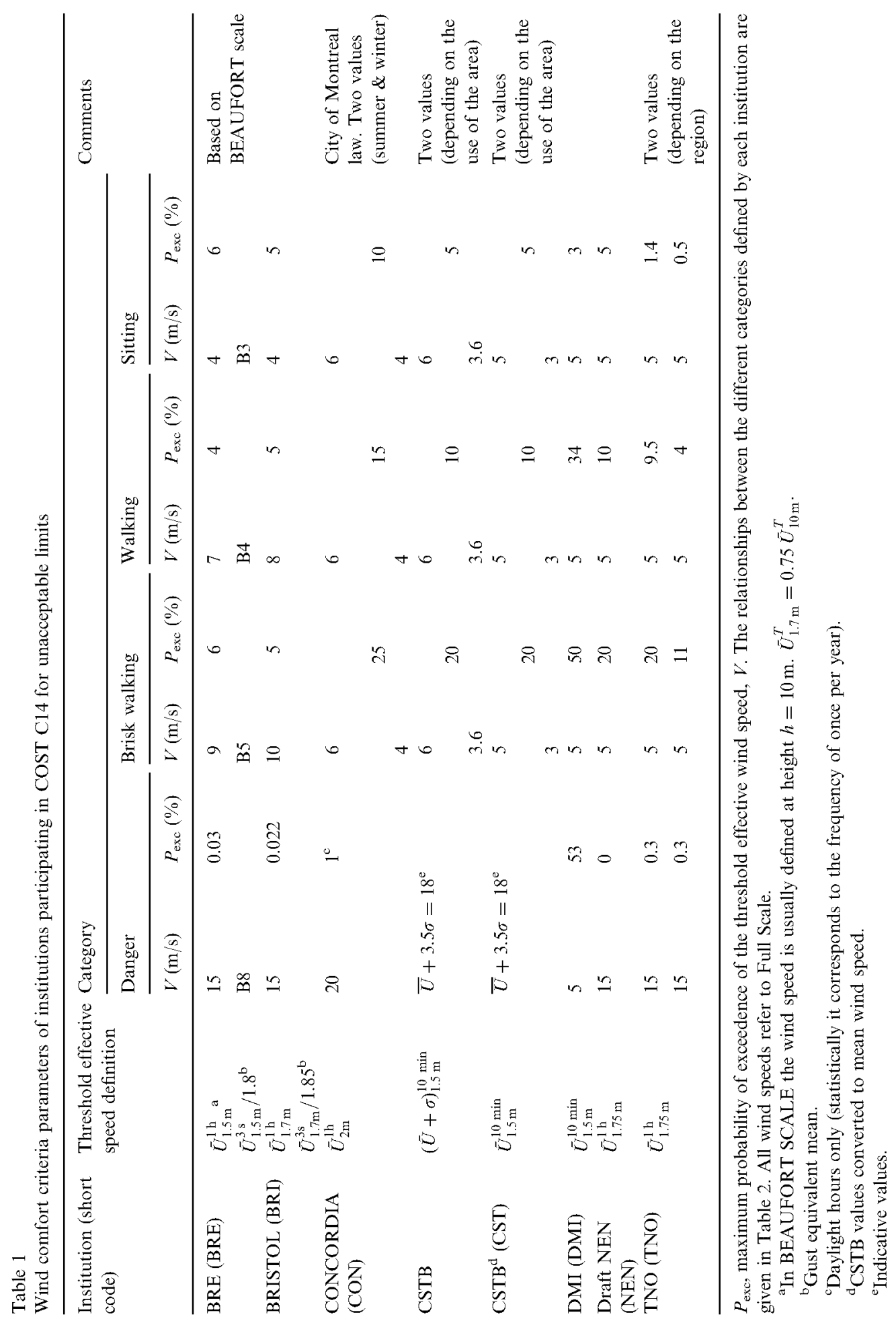


definition Koss, 2004, there are two types of criteria: a) the ones based on the mean wind speed and b) those based on a kind of gust speed. The mean wind speed at a given point around a building (local wind speed) should be proportional to the incident or climate wind speed. Two type-a criteria applied to the same point will consider the same climate wind speed multiplied by the same amplification factor. Therefore the local wind speed probability distribution will have a shape like the climatic wind speed probability distribution (see Section 3) and this probability distribution can be employed to compare the two criteria. This statement cannot be applied to the type-b criteria because the local behaviour of the turbulence could affect in a different way the probability distribution to be considered in each criteria. However, in order to allow a first comparative work we will substitute the gust speed by an equivalent mean speed. The type-a threshold effective wind speed is defined here as $\bar{U}_{h}^{T}$ where $T$ is the averaging interval, and $h$ is the height where the speed is measured.

Several assumptions have been taken into account in building up Table 1 , in order to simplify the criteria classification:

1. Just one figure (instead of a range of values) is employed to define a limit when criteria based on the BEAUFORT scale are considered.

2. Values are rounded off to integer numbers (where possible).

3. Only the "unacceptable" limit is being considered.

4. As different institutions define different categories, an attempt has been made to reduce them just to four (the most used): danger (D), brisk walking (B), walking (W) and sitting (S). The correspondence among the categories defined by the institutions and the ones defined here is shown in Table 2 .

\subsection{Corrections for averaging time and height reference}

In addition to different categories, definitions of the threshold effective wind speed employed by the institutions are also different, because they are based on different values of the averaging time ( $1 \mathrm{~h}$ or $10 \mathrm{~min})$ and of the height reference $(1.5-2 \mathrm{~m})$. For height reference conversion a relationship $\bar{U}_{1.7 \mathrm{~m}}^{T}=0.75 \bar{U}_{10 \mathrm{~m}}^{T}$ can be generally assumed. Concerning time reference conversion, according to Joint Committee on Structural Safety (2001) the following relationships can be applied

$$
1.05 \bar{U}^{1 \mathrm{~h}}=1.0 \bar{U}^{10 \min }=0.84 \bar{U}^{1 \mathrm{~min}}=0.67 \bar{U}^{3 \mathrm{~s}}
$$

in open terrain exposure. Although the differences introduced by conversion to a common reference would not be always small, they are not corrected for in the present study as the main aim is to analyze the comparison feasibility. In the case of conversion between $10 \mathrm{~min}$ and $1 \mathrm{~h}$ values, which are the most common definitions, just a 5\% correction would be needed. Anyway, the definition of the threshold speed (in terms of averaging time and height reference) should be always clearly stated, in order to allow for a correct comparison exercise.

\section{Comparison method}

A given limit criteria can be summarized by specifying both the threshold effective speed value $V$ (in $\mathrm{m} / \mathrm{s}$ ) and the probability of excedence, $P_{\text {exc }}$. In this paper the notation 
Table 2

Wind comfort criteria. Relationships assumed in Table 1 between the different categories defined by each institution

\begin{tabular}{|c|c|c|c|c|c|}
\hline \multirow[t]{2}{*}{ Institution } & \multicolumn{5}{|l|}{ Category } \\
\hline & $\mathrm{D}$ & B & W & $\mathrm{S}$ & $\begin{array}{l}\text { Intermediate } \\
\text { category }\end{array}$ \\
\hline BRE & Danger & $\begin{array}{l}\text { Objective } \\
\text { "business" } \\
\text { walking }\end{array}$ & $\begin{array}{l}\text { Pedestrian } \\
\text { walking }\end{array}$ & Sitting & $\begin{array}{l}\text { Workers round } \\
\text { buildings? }\end{array}$ \\
\hline BRISTOL & Distress levels & Business walking & $\begin{array}{l}\text { Pedestrian } \\
\text { walking }^{\mathrm{a}}\end{array}$ & Pedestrian sitting & Pedestrian standing \\
\hline $\begin{array}{l}\text { Concordia } \\
\text { Univ. }\end{array}$ & Danger & $\begin{array}{l}\text { Main streets/ } \\
\text { secondary streets }\end{array}$ & $\begin{array}{l}\text { Main streets/ } \\
\text { Secondary streets } \\
\text { /parks }\end{array}$ & Parks & N.A. \\
\hline CSTB & Danger & Brisk walking & $\begin{array}{l}\text { Pedestrian } \\
\text { walking }\end{array}$ & Steady position ${ }^{a}$ & $\begin{array}{l}\text { Very long steady } \\
\text { position }\end{array}$ \\
\hline DMI & Danger & Quick walk & Slow walk ${ }^{a}$ & $\begin{array}{l}\text { Standing or } \\
\text { sitting for a long } \\
\text { time }\end{array}$ & $\begin{array}{l}\text { Standing or sitting } \\
\text { for a short time }\end{array}$ \\
\hline Draft NEN & Danger & Traversing area & Lounging area & $\begin{array}{l}\text { Sitting and } \\
\text { standing }\end{array}$ & N.A. \\
\hline $\mathrm{TNO}$ & Danger & $\begin{array}{l}\text { Pedestrian } \\
\text { walking }\end{array}$ & Lounging area & $\begin{array}{l}\text { Sitting and } \\
\text { standing }\end{array}$ & N.A. \\
\hline
\end{tabular}

N.A.: not applicable.

${ }^{\text {a } I n t e r m e d i a t e ~ c a t e g o r y ~ p o s i t i o n . ~}$

$\left(V, P_{\mathrm{exc}} \times 100\right)$ is used. The points of the $\left(V, P_{\mathrm{exc}}\right)$ plane, which represent some of the criteria among those defined in Table 1 , are plotted in Fig. 1, together with some representative exceedence probability Weibull functions. It is clear that a given probability distribution can satisfy several comfort criteria.

More than 30 points should have been included in Fig. 1 if all criteria reflected in Table 1 would have been taken into account. In order to simplify the presentation in Fig. 1 a first attempt to reduce the number of criteria has been made by: 1) excluding the Danger Category, and 2) joining those criteria that are close to each other (CST, NEN, TNO) and labelling them with the same code, as shown in Table 3 (the same code as used in Fig. 1). A smaller set, containing 21 points only, are presented as a result of this reduction exercise.

As it can be shown in Fig. 1 and (Koss, 2004), it is difficult to establish a comparison among the different criteria, because of the two-dimensional character (speed and probability) of the criteria definition. The problem is the same as trying to identify some ascending or descending order in a set of points placed in a two-dimensional geometrical plane. This is the reason why it is difficult to say which criterion is more restrictive than other (in the sense that it is reached first), or even to try to compare them in general.

Concerning the comparison exercise, the probabilistic description of the wind as a Weibull probability function can help. In fact, as shown in the wind tunnel experiments performed by Pearce and Baker (1999) the probability distribution of instantaneous local wind speed at points placed in an urban environment can be described by a Weibull distribution (for speeds larger than the mean value), in the same way than the mean meteorological wind speed. Moreover, Wisse and Willemsen (2003) present an 




Fig. 1. Wind comfort criteria. Individual points represent wind comfort criteria for unacceptable limits, defined as the maximum probability of exceedence, $P_{\text {exc }}$, of a given threshold effective wind speed, $V(\mathrm{~m} / \mathrm{s})$. Symbols: starsbrisk walking, circle-walking; $x$-sitting. The figures close to the symbols denote the institution criteria, according to the code established in Table 3. Also (shown for reference) there are several wind climates represented by Weibull exceedence probability functions $P_{\text {exc }}$ (solid curves) according to expression (3), for $k=1.5$ and $c$ is given by the figures close to the solid curves. Each curve represents a wind climate.

Table 3

Identification codes for criteria used in Fig. 1

\begin{tabular}{llcl}
\hline Institution & \multicolumn{2}{c}{ Category } & \\
\cline { 2 - 4 } & $\mathrm{B}\left({ }^{*}\right)^{\mathrm{a}}$ & $\mathrm{W}(0)^{\mathrm{a}}$ & $\mathrm{S}(\times)^{\mathrm{a}}$ \\
\hline BRE & $1(9,6)$ & $9(7,4)$ & $17(4,6)$ \\
BRI & $2(10,5)$ & $10(8,5)$ & $17(4,5)$ \\
CON (a) & $3(6,25)$ & $11(6,15)$ & $18(6,10)$ \\
CON (b) & $4(4,25)$ & $12(4,15)$ & $19(4,10)$ \\
CST (a) & $5(5,20)$ & $13(5,10)$ & $15(5,5)$ \\
NEN & & & $20(5,1)$ \\
TNO (a) & $6(3,20)$ & $14(3,10)$ & $21(3,5)$ \\
CST(b) & $7(5,11)$ & $15(5,4)$ & $20(5,0,5)$ \\
TNO (b) & $8(5,50)$ & $16(5,34)$ & $15(5,3)$ \\
DMI & & &
\end{tabular}

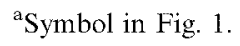

experimental relation between threshold effective wind speed $V$ and probability of exceedence $P_{\text {exc }}$ derived from wind speed ratios for a set of building plans that also shows this trend. The Weibull probability density function $f(x)$ is defined as follows:

$$
f(x)=\frac{k}{c}\left(\frac{x}{c}\right)^{k-1} \exp \left[-\left(\frac{x}{c}\right)^{k}\right]
$$


where $k$ and $c$ are the shape and scale parameters, respectively, which are related to the mean value and the standard deviation in the following way

$$
\bar{x}=c \Gamma\left(1+\frac{1}{k}\right) ; \quad \sigma^{2}=c^{2}\left[\Gamma\left(1+\frac{2}{k}\right)-\Gamma^{2}\left(1+\frac{1}{k}\right)\right]
$$

where $\Gamma$ is the gamma function (Marchante et al., 2000). Some characteristic values of the Weibull probability distribution are shown in Table 4 . The Weibull cumulative distribution function is given by

$$
F(x<V)=1-\exp \left[-\left(\frac{V}{c}\right)^{k}\right], \quad 0<V<\infty ; \quad c>0, \quad k>0 .
$$

As shown in Table 4 increasing $c$ means larger mean speeds, and larger $k$ means lower standard deviation. The probability that the wind speed exceeds a given limit $V$ associated to some specific wind climate (whose characteristics are defined by some specific values $k$ and $c)$ is given by $P_{\text {exc }}(V)$ as follows:

$$
P_{\text {exc }}(V)=1-F(x<V)=\exp \left[-\left(\frac{V}{c}\right)^{k}\right]
$$

A specific wind climate is associated to an exceedence probability function, defined by specific values $k$ and $c$. As shown in Fig. 1 a specific wind climate $(c, k)$ can fulfil several criteria, and the vice versa is also true.

In order to clarify this situation, let us define a given limit criterion " $i$ ", by a couple of numbers $\left(V_{i}, P_{\text {exc }} i\right)$. Therefore all wind conditions $(c, k)$, that is, all the exceedence probability distributions functions (3) that satisfy this criterion must fulfil the relationship that is obtained by introducing the values $\left(V_{i}, P_{\operatorname{exc} i}\right)$ in (3), as follows

$$
P_{\text {exc } i} \equiv P_{\text {exc }}\left(V_{i}\right)=\exp \left[-\left(\frac{V_{i}}{c}\right)^{k}\right]
$$

By taking logarithms twice, one obtains

$$
k=\frac{\ln \left[-\ln \left(P_{\operatorname{exc} i}\right)\right]}{\ln \left(V_{i} / c\right)}
$$

This relationship defines a line $k\left(c ; V_{i}, P_{\operatorname{exc} i}\right)$ in the $(c, k)$ plane that is the locus of all wind probability distributions (let say wind climates) that fulfil a given criterion $\left(V_{i}, P_{\operatorname{exc} i}\right)$. This line is called iso-criteria line. However, the expression (4a) is difficult to appraise, but it can

Table 4

Some characteristic values of the Weibull probability distribution.

\begin{tabular}{llll}
\hline$k$ & $\bar{x} / c$ & $\sigma^{2} / c^{2}$ & $\sigma / \bar{x}$ \\
\hline $1 / 2$ & 2 & 20 & $\sqrt{5}$ \\
1 & 1 & 1 & 1 \\
2 & $\sqrt{\pi} / 2$ & $1-\pi / 4$ & $\sqrt{(4-\pi) / \pi}$ \\
\hline
\end{tabular}

$\bar{x}$ : mean; $\sigma:$ standard deviation; $k$ : shape parameter; $c$ : scale parameter. 
be rewritten in the following way

$$
\frac{1}{k}=\frac{1}{\ln \left[-\ln \left(P_{\mathrm{exc} i}\right)\right]}\left[\ln \left(V_{i}\right)-\ln (c)\right]=p\left[\ln (c)-\ln \left(V_{i}\right)\right]
$$

Expression (4b) shows that $1 / k$ is a linear function of $\ln (c)$, whose slope $p=-1 / \ln \left[-\ln \left(P_{\text {exc } i}\right)\right]$ depends only on the probability of exceedence, cutting the abscissa axis at $\ln (c)=\ln \left(V_{i}\right)$. In this way, each criterion is associated to one straight line in the $\left(\ln (c), k^{-1}\right)$ plane, whose geometrical characteristics (slope and $x$-axis crossing abscissa) are clearly and independently related to the criteria definition parameters $\left(V_{i}, P_{\text {exc }} i\right)$. On the other hand, similar or equivalent criteria are those whose associated iso-criteria lines are close to each other.

Even with this new approach, as the number of criteria is too large, the large amount of curves in the plane corresponding to all the criteria in Table 3 would obscure the comparison. To get rid of this problem, a second grouping and reduction of criteria has been performed, as shown in Table 5. As can be observed by comparison of both tables, the eight rows in Table 3 are reduced to just three: cases b) of CON, CST, TNO and DMI are not included (for the shake of simplicity in the presentation), and BRE and BRI are put together in the same place. The iso-criteria lines associated to the criteria $\left(V_{i}, P_{\text {exc }}\right)$ displayed in Table $5(i=1-9)$ are shown in Figs. 2 and 3, both considered as functions $k$ $\left(c ; V_{i}, P_{\text {exc } i}\right)$ defined by (4a) and considered as functions $1 / k\left(\ln c ; V_{i}, P_{\text {exc } i}\right)$ defined by (4b). The criteria $i=1,2,3$ correspond to category $\mathrm{B}$, criteria $i=4,5,6$ correspond to category $\mathrm{W}$, and criteria $i=7,8,9$ correspond to category $\mathrm{S}$. Criteria are displayed by contiguous categories, that is B and W in Fig. 2, and W and S in Fig. 3 for easy comparison, instead of plotting B, W and S together in the same figure. In Figs. 2 and 3 the upper graphs show a direct appraisal of the $c$ and $k$ values in each point, whilst in the lower ones, $1 / k=f(\ln c)$, the relative position of the limits are both more clearly defined and understandable.

The criteria lines can be referred to as (X, YY) by using the code defined in Table 5 (X stands for category and YY for institution). For instance, the iso-criteria line associated to criterion 3 can be denoted as (B, CT). We will say that complete order between criteria exists if there is no intersection of lines (in the range of interest), and partial order otherwise.

As shown in Fig. 2, in the B category the criterion 3 (B, CT) is the most restrictive, as it is placed in the leftist place, when $k>1$ that is most often the case. Compared with each other, the criteria 1 and 2 show a partial order because the associated curves have an intersection at roughly $c=5$. Below $c=5$ (relative low speeds) criterion $1(\mathrm{~B}, \mathrm{BB})$ is more

Table 5

Identification codes for criteria used in Figs. 2 and 3

\begin{tabular}{lllll}
\hline Institutions (short code) & \multicolumn{2}{l}{ Category } & \\
\cline { 3 - 5 } & & $\mathrm{B}$ & $\mathrm{W}$ & $\mathrm{S}$ \\
\hline BRE & & $1(9,6)$ & $4(7,4)$ & $7(4,6)$ \\
BRI & (BB) & & & \\
CON (a) & (CO) & $2(6,25)$ & $5(6,15)$ & $8(6,10)$ \\
CST (a) NEN & & $3(5,20)$ & $6(5,10)$ & $9(5,5)$ \\
TNO (a) & (CT) & & &
\end{tabular}

Unacceptable limits, according to the nomenclature $\left(V_{i}[\mathrm{~m} / \mathrm{s}], P_{\text {exc } i} \times 100\right)$. 

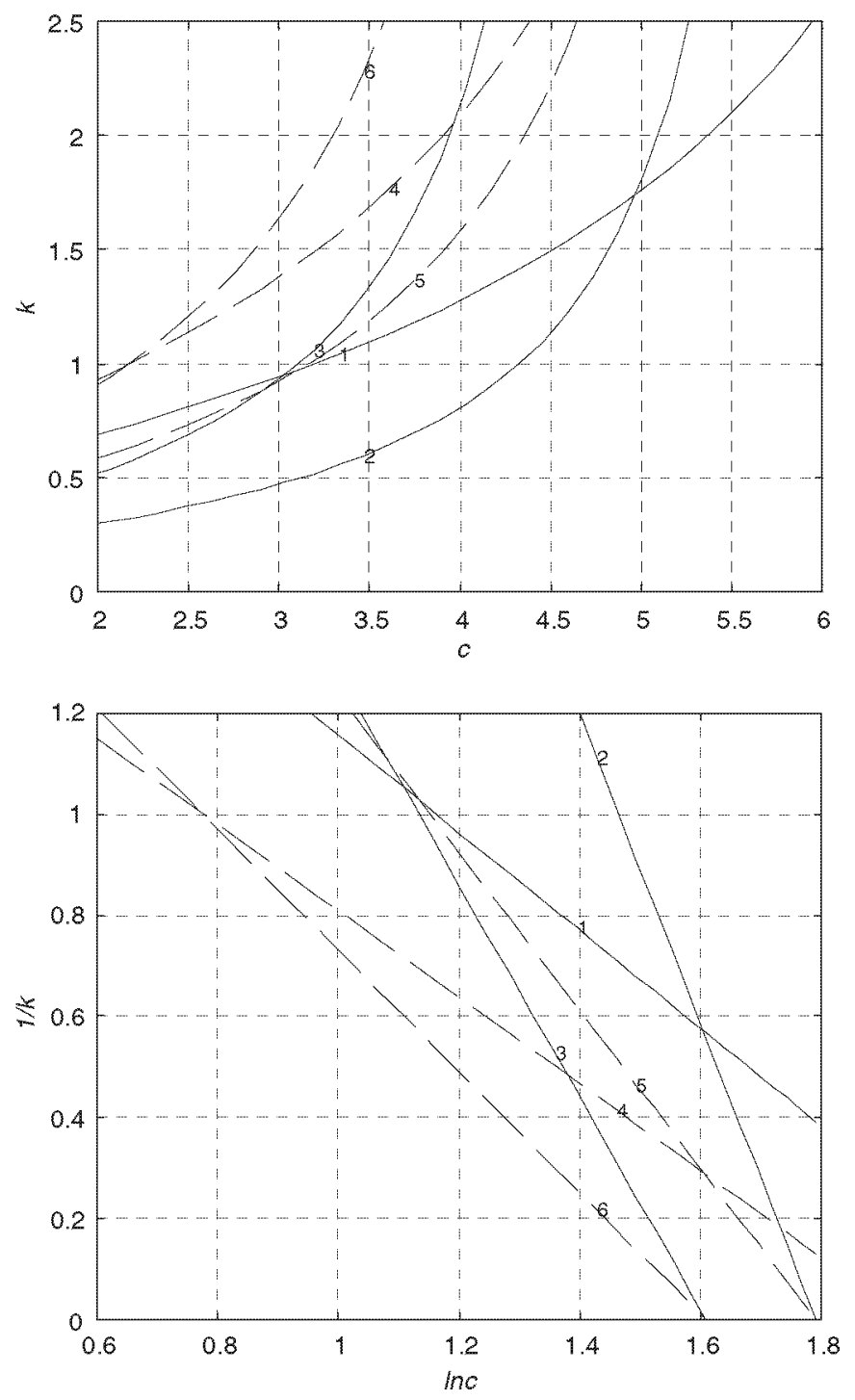

Fig. 2. Iso-criteria lines for some wind comfort criteria for unacceptable limits. Scale and shape parameters, $c$ and $k$, respectively, of the Weibull probability distributions (according to Eq. (4)) that matches the wind comfort criteria defined in Table 5. The categories B (brisk walking, solid lines) and $\mathrm{W}$ (walking, dashed lines) are considered.

restrictive than criterion $2(\mathrm{~B}, \mathrm{CO})$, and vice versa. It is interesting to notice that the most restrictive criterion, $3(\mathrm{~B}, \mathrm{CT})$, is proposed by three western European continental based laboratories. Then criteria 1 and 2 are proposed by the laboratories located in countries where the climatic conditions are either windy (UK) or colder (Canada). It is interesting to underline that for higher wind velocity criterion 2 is more restrictive than criterion 1 . This may also be due to the influence of thermal comfort parameters that interfere with wind comfort parameters (low temperature becoming more critical with higher wind velocities). 

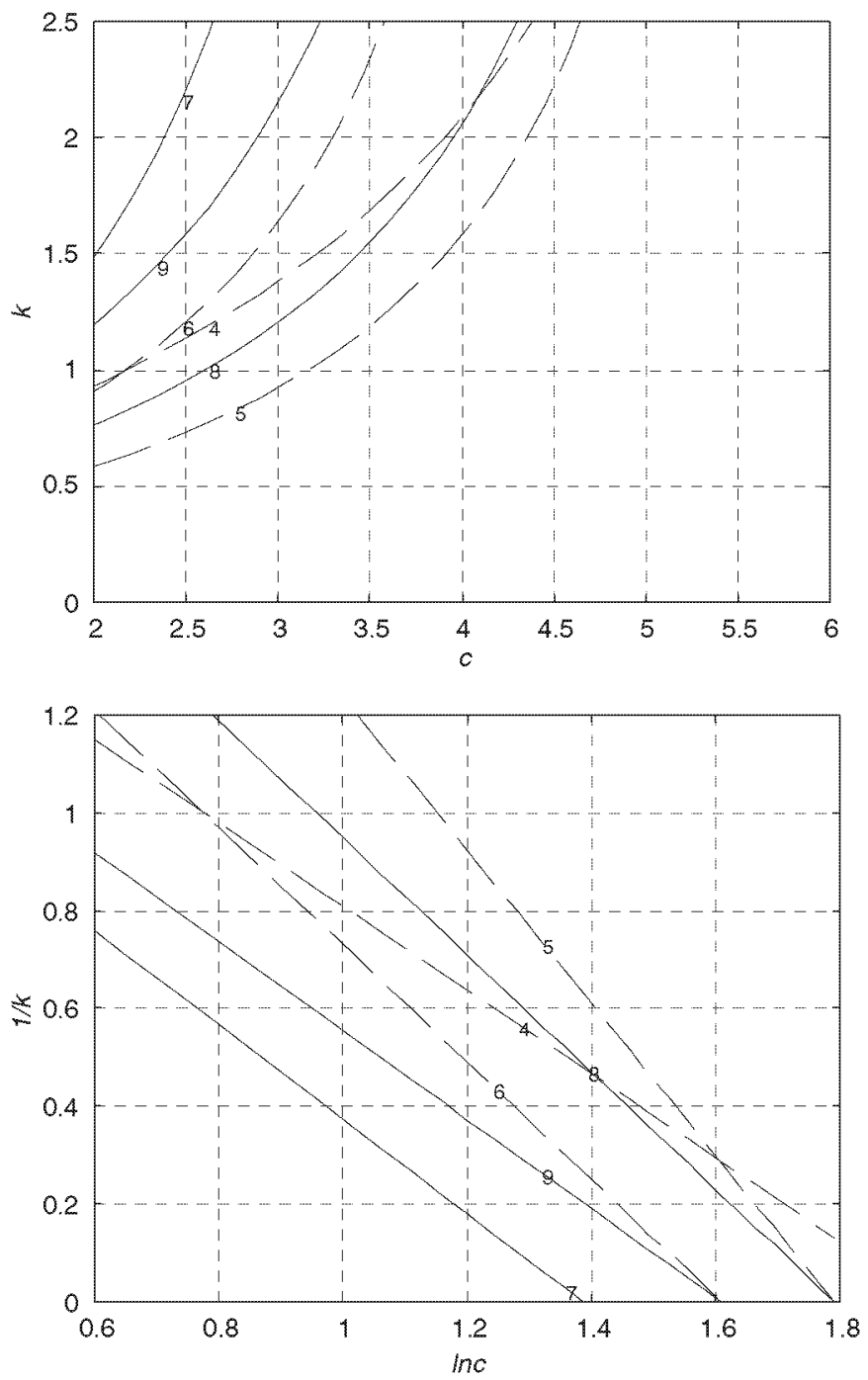

Fig. 3. Iso-criteria lines for some wind comfort criteria for unacceptable limits. Scale and shape parameters, $c$ and $k$, respectively, of the Weibull probability distributions (according to Eq. (4)) that matches the wind comfort criteria defined in Table 5. The categories $\mathrm{W}$ (walking, dashed lines) and S (sitting, solid lines) are considered.

In the category $\mathrm{W}$ complete order exits, and the criteria, sorted by restriction, are 6 $(\mathrm{W}, \mathrm{CT}), 4(\mathrm{~W}, \mathrm{BB})$ and finally $5(\mathrm{~W}, \mathrm{CO})$. However, there is not complete order among criteria in categories $\mathrm{B}$ and $\mathrm{W}$, as the limit $3(\mathrm{~B}, \mathrm{CT})$ is more restrictive than $5(\mathrm{~W}, \mathrm{CO})$ and even than $4(\mathrm{~W}, \mathrm{BB})$ when $c>4$.

It is very easy to identify these details in the lower graph, $1 / k=f(\ln c)$, because the relative position of the straight lines is clearly visible. In addition, observe that criteria with the same threshold velocity $V_{i}$ cuts the abscissa axis at the same point $\left(\ln V_{i}, 0\right)$, and criteria with the same probability of exceedence are parallel to each other. 
As shown in Fig. 3, in the category $\mathrm{S}$ (criteria 7, 8 and 9) the order is 7, 9, 8, taken from more restrictive to less restrictive. The order is complete because there is no intersection of iso-criteria lines. No complete order exists among categories $\mathrm{W}$ and $\mathrm{S}$ because criteria 6 and 4 (only if $c>4$ ) appear to be more restrictive than 8 , that is, walking limit by BB and CT (criteria 4 and 6) are more restrictive than sitting limit by $\mathrm{CO}$. In all categories considered the criteria from $\mathrm{CO}$ (criteria 2, 5 and 8) seems to be less restrictive than the others.

The method here presented has been applied just to a few criteria, but it could be also used to compare the remaining criteria and determine their relative restrictiveness.

\section{Practical use}

Usually the parameters of the Weibull distribution of the climatic wind, $k_{\mathrm{c}}(\theta)$ and $c_{\mathrm{c}}(\theta)$, are known from wind speed meteorological data in several wind directions, $\theta_{i}$. It is possible to make estimations of appropriate mean values of $k_{\mathrm{c}}$ and $c_{\mathrm{c}}$, in order to use them for comparison with the criteria, by using Figs. 2 and 3 , as shown below.

The wind speed at a given point $P$ influenced by a building, in terms of the mean wind speed at head height $h h, U_{h h P}^{10 \mathrm{~min}}$, when the wind direction is $\theta_{i}$, can be expressed as follows

$$
U_{h h P}^{10 \min }=G_{u i}^{P} U_{10 \mathrm{~m}}^{10 \mathrm{~min}},
$$

where $G_{u i}^{P}=G_{u}^{P}\left(\theta_{i}\right)$ is the amplification at point $P$ of the mean meteorological speed (let say $U_{10 \mathrm{~m}}^{10 \mathrm{~min}}$ ) produced by the building when the wind direction is $\theta_{i}$. Then the probability that the local wind speed at head height at point $P, U_{h h P}^{10 \mathrm{~min}}$, will exceed some limit $V$ is the same that the mean wind speed will exceed $V / G_{u i}^{P}$

$$
P_{\text {exc }}\left(V, \theta_{i}\right)=\exp \left[-\left(\frac{V}{G_{u i}^{P} c_{\mathrm{c} i}}\right)^{k_{\mathrm{c} i}}\right]
$$

where $c_{\mathrm{c} i}=c_{\mathrm{c}}\left(\theta_{i}\right), k_{\mathrm{c} i}=k_{\mathrm{c}}\left(\theta_{i}\right)$ are the Weibull parameters for each climatic wind direction $\theta_{i}$. The probability of exceedence of a given value $V, P_{\text {exc }}(V)$, is the addition of the contributions of $N$ directions

$$
P_{\text {exc }}(V)=\sum_{i=1}^{N} P\left(\theta_{i}\right) \exp \left[-\left(\frac{V}{G_{u i}^{P} c_{\mathrm{c} i}}\right)^{k_{c i}}\right],
$$

where $P\left(\theta_{i}\right)$ is the probability of each wind direction $\theta_{i}$. By using

$$
k_{\mathrm{c} i}=\bar{k}_{P}\left(1+\varepsilon_{i}\right) ; \quad c_{\mathrm{c} i} G_{u i}^{P}=\bar{c}_{P}\left(1+\delta_{i}\right),
$$

it can be shown (see the Appendix) that the total exceedence probability $P_{\text {exc }}(V)$ is given by

$$
P_{\text {exc }}(V)=\mathrm{e}^{-\left(\frac{V}{c_{P}}\right)^{\bar{k}_{P}}},
$$

where

$$
\bar{c}_{P}=\sum_{i=1}^{N} G_{u i}^{P} c_{\mathrm{c} i} P\left(\theta_{i}\right) ; \quad \bar{k}_{P}=\sum_{i=1}^{N} k_{\mathrm{c} i} P\left(\theta_{i}\right),
$$

if the conditions $\delta_{i}, \varepsilon_{i} \ll 1$ are fulfilled. Eq. (9) shows that the total exceedence probability distribution of the wind at point $P$ obeys a Weibull distribution whose parameters $c=\bar{c}_{P}$ 


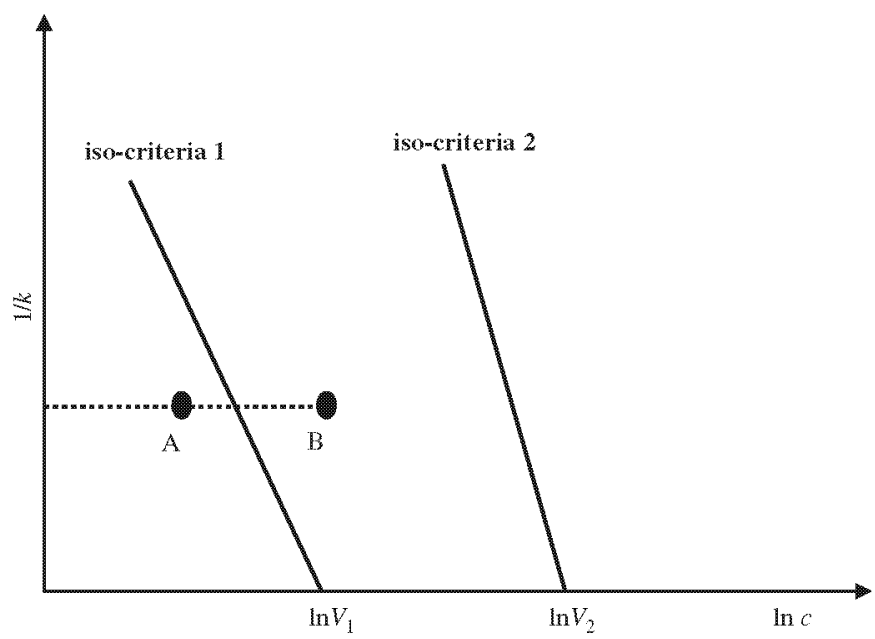

Fig. 4. Wind comfort criteria comparison diagram. Scale and shape parameters, $c$ and $k$, respectively, of the Weibull probability distributions (according to Eq. (4b)). $V_{1}$ and $V_{2}$ are the threshold effective wind speeds of criteria 1 and 2, respectively. The position of an equivalent local mean wind (A or B) with regard to the iso-criteria line 1 shows the degree of fulfilment of the criterion: fulfilment on the left (A), unfulfilment on the right (B). Criteria 2 is less restrictive than criteria 1.

and $k=\bar{k}_{P}$ are weighted averages of the directional parameters $k\left(\theta_{i}\right)$ and $c\left(\theta_{i}\right)$. The amplification factor $G_{u i}^{P}$ can be measured on an urban scale model by appropriated wind tunnel testing. Observe that the parameter $\bar{k}_{P}$ is not influenced by the building. $\bar{k}_{P}$ is just a combination of the climatic values $k_{\mathrm{c} i}$. The amplification produced by the building affects only to the value of $\bar{c}_{P}$. Therefore, $\bar{k}_{P}$ is the same for the total exceedence probability distributions at all points, while $\bar{c}_{P}$ changes from on point to another due to the different values of the amplification factor.

It can be considered that the values $c=\bar{c}_{P}$ and $k=\bar{k}_{P}$ represents the total exceedence probability distribution of an "equivalent local mean wind" in order to evaluate the pedestrian comfort. In fact, once $\bar{c}_{P}$ and $\bar{k}_{P}$ are determined from (10) for a given point $P$ in an urban environment, they can be plotted as a point $\left(\bar{c}_{P}, \bar{k}_{P}\right)$ in graphs $(c, k)$ of Figs. 2 or 3 in order to observe its position with regard to the limits associated to the different criteria. In this way the degree of fulfilment of the relevant criteria can be determined (fulfilment on the left, unfulfilment on the right of the relevant limit, see Fig. 4). The comfort degree could be defined as the distance from the "equivalent local mean wind" $\left(\bar{c}_{P}, \bar{k}_{P}\right)$ point to the iso-criteria line in graphs $(c, k)$. In a practical situation, as $\bar{k}_{P}$ is the same for all the points around the building, the actions taken to increase the comfort, moving the point to the left (by reducing the amplification factor) will only move the point $\left(\bar{c}_{P}, \bar{k}_{P}\right)$ along the horizontal, and therefore the distance to the iso-criteria line can only be modified in the horizontal direction.

\section{Conclusions}

A method that allows the comparison of the several existing wind comfort criteria, developed and being used by COST C14 members, has been presented as a first step in the process of definition of a common methodology. Some assumptions have been made and a 
grouping of criteria has been performed to simplify the definition of the existing criteria as a first step to reduce the complexity of the comparison. The method presented here is applied as an example to the abovementioned groups of criteria and their relative restrictiveness are shown.

The differences in the criteria used by each laboratory appear because, among other circumstances, they are not necessarily all based on the same concept of pedestrian comfort. In colder, hotter, windier, etc. climates there could also be an element of human perception or acclimatisation built into the comfort criteria. For example the criteria used in UK are for inland areas in the south of the UK, when a site in the North of the country or on the coast is analysed some changes in the threshold conditions are considered to account for the acclimatisation/perception of the local population to these local conditions. This could be part of the reason for the differences between laboratories. Anyway, in order to clarify the origin of these differences and to reach the COST C14 objectives, some actions should be accomplished:

1. The study of the criteria based on the gust wind speed and the analysis of their relationship with criteria based on mean wind speeds (which are more convenient to apply).

2. Once the comparison between criteria is made possible, the aim is the search for real information (in terms of comfort), which is responsible for the differences between criteria developed by the institutions.

3. The study of the definition of common criteria, which likely would require regional or climatic adaptations.

The comparison performed here is mainly considered as a formal and mathematical exercise, and it should be taken as valid for a given wind speed range, because the modelization of the wind speed probability distribution by Weibull function has its own limitations.

\section{Acknowledgements}

The authors wish to deeply acknowledge the collaboration and constructive criticism of the members involved on WG1 (C. J. Baker, P. A. Blackmore, E. Canepa, P. Delpech, C.P.W. Guerts, H. Koss, T. Stathopoulos, E. Willemsen, J.A. Wisse) and the support offered by COST C14 action.

\section{Appendix}

The probability of exceedence is

$$
P_{\text {exc }}=\sum_{i=1}^{N} P\left(\theta_{i}\right) \mathrm{e}^{-\left(\frac{x}{c}\right)^{k_{i}}}
$$

Let us consider each term expanded in Taylor series for small values of $\delta_{i}, \varepsilon_{i}$, defined as follows

$$
\mathrm{e}^{-\left(\frac{x}{c_{i}}\right)^{k_{i}}}=\mathrm{e}^{-\left(\frac{x}{c\left(1+\delta_{i}\right)}\right)^{\bar{k}\left(1+\varepsilon_{i}\right)}} \simeq f_{c} \bar{c} \delta_{i}+f_{\mathrm{k}} \bar{k} \varepsilon_{i}+\mathrm{e}^{-\left(\frac{x}{\bar{c}}\right)^{\bar{k}}}
$$


where

$$
\begin{aligned}
f_{\mathrm{c}} & =\frac{\partial}{\partial c}\left[\mathrm{e}^{-\left(\frac{x}{c}\right)^{k}}\right] ; \quad f_{\mathrm{k}}=\frac{\partial}{\partial k}\left[\mathrm{e}^{-\left(\frac{x}{c}\right)^{k}}\right], \\
c_{i} & =\bar{c}\left(1+\delta_{i}\right) \rightarrow \delta_{i}=\frac{c_{i}-\bar{c}}{\bar{c}} ; \quad \varepsilon_{i}=\frac{k_{i}-\bar{k}}{\bar{k}} \quad \text { and } \quad \delta_{i}, \varepsilon_{i} \ll 1 .
\end{aligned}
$$

By substitution in (A.1)

$$
\begin{aligned}
P_{\mathrm{exc}} & \left.\left.=\sum_{i=1}^{N} P\left(\theta_{i}\right) \delta_{i}\right) f_{\mathrm{c}} \bar{c}+\sum_{i=1}^{N} P\left(\theta_{i}\right) \varepsilon_{i}\right) f_{\mathrm{k}} \bar{k}+\mathrm{e}^{-\left(\frac{x}{\bar{c}}\right)^{\bar{k}}} \\
& =\delta \bar{c} f_{\mathrm{c}}+\varepsilon \bar{k} f_{\mathrm{k}}+\mathrm{e}^{-\left(\frac{x}{\bar{c}}\right)^{\bar{k}}} \simeq \mathrm{e}^{-\left(\frac{x}{\bar{\alpha}(1+\delta)}\right)^{\bar{k}(1+\delta)}}
\end{aligned}
$$

$\bar{c}$ and can be obtained by using the conditions $\delta=\varepsilon=0$. In the case of $\delta$

$$
\begin{aligned}
\delta & =\sum_{i=1}^{N} P\left(\theta_{i}\right) \delta_{i}=\sum_{i=1}^{N}\left(\frac{c_{i}-\bar{c}}{\bar{c}}\right) P\left(\theta_{i}\right) \\
& =\sum_{i=1}^{N} \frac{c_{i}}{\bar{c}} P\left(\theta_{i}\right)-\sum_{i=1}^{N} P\left(\theta_{i}\right)=\sum_{i=1}^{N} \frac{c_{i}}{\bar{c}} P\left(\theta_{i}\right)-1,
\end{aligned}
$$

and by using $\delta=0$

$$
\bar{c}=\sum_{i=1}^{N} c_{i} P\left(\theta_{i}\right),
$$

In a similar way

$$
\bar{k}=\sum_{i=1}^{N} k_{i} P\left(\theta_{i}\right),
$$

and therefore

$$
P_{\text {exc }}(x)=\mathrm{e}^{-\left(\frac{x}{\bar{c}}\right)^{\bar{k}}}
$$

where $\bar{c}$ and $\bar{k}$ are defined by (A.6) and (A.7). 


\section{References}

Davenport, A.G., 1972. An approach to human comfort criteria for environmental wind conditions. Colloqiom. on Building Climatology.

Gandemer, J., 1975. Wind environment around buildings: aerodynamic concept. In: Proceedings of the Fourth International Colloquium on Wind Effects on Buildings and Structures, Heathrow.

Isyumov, N., Davenport, A.G., 1975. The ground level wind environment in built-up areas.In: Proceedings of the Fourth Colloquium on Wind Effects on Buildings and Structures, Heathrow.

Joint Committee on Structural Safety (JCSS), 2001. Probabilistic Model Code. Part 2: Loads, Chapter 2.13, 〈www.jcss.ethz.ch〉.

Koss, H., 2004. On differences and Similarities on applied wind comfort criteria. In: Proceedings of the International Conference on Urban Wind Engineering and Building Aerodynamics. VKI, Brussels, pp. B.2.1-B.2.11.

Marchante, M., Martí, I., Navarro, J., García, C., 2000. Estimating the Weibull parameters using two different methodologies. In: Proceedings of the European Wind Energy Conference, Special Topic, Kassel.

Melbourne, W.H., 1978. Criteria for environmental wind conditions. J. Wind Eng. Ind. Aerodyn. 3.

Pearce, W., Baker, C.J., 1999. Wind tunnel tests on the dispersion of vehicular pollutants in an urban area. J. Wind Eng. Ind. Aerodyn. 80 (3), 327-349.

Ratcliff, M.A., Peterka, J.A., 1990. Comparison of pedestrian wind acceptability criteria. J. Wind Eng. Ind. Aerodyn. 36.

Wisse, J.A., Willemsen, E., 2003. Standardization of wind comfort evaluation in the Netherlands, In: Proceedings of the 11th ICWE, Lubbock. 